J. Lake Sci. (湖泊科学), 2015, 27(2): 327-334

http: //www. jlakes. org. E-mail : jlakes@niglas.ac.cn

(c) 2015 by Journal of Lake Sciences

\title{
离散型湖泊水体提取方法精度对比分析”
}

\author{
吉红霞 ${ }^{1,2}$, 范兴旺 ${ }^{1,2}$, 吴桂平 ${ }^{1}$, 刘元波 ${ }^{1 * *}$ \\ ( 1 : 中国科学院南京地理与湖泊研究所,南京 210008) \\ (2: 中国科学院大学,北京 100049 )
}

\begin{abstract}
摘 要: 基于卫星遥感的陆地水体提取方法多种多样, 并且应用广泛. 对于水体分布支离破碎的枯水期湖泊, 准确的水体 提取方法尚不明晰, 直接影响湖泊水域面积的提取精度. 以鄱阳湖湖区为研究对象, 利用 ALOS 遥感影像, 以 $2.5 \mathrm{~m}$ 高分 辨率全色波段融合影像非监督分类 (ISODATA) 得到的水体面积为参考值, 分别使用归一化水体指数 (NDWI) 法、NDWIISODATA 法和基于近红外 (NIR) 的 ISODATA 法提取了 $10 \mathrm{~m}$ 分辨率的水体分布, 分析了不同方法提取结果之间的差异性 及产生原因. 结果表明: 3 种方法均可以较好地提取出水体, 但利用 ISODATA 法提取的水体细部信息更为明显, 面积值较 $N D W I$ 法更大; 相对于近红外单波段而言, 基于 $N D W I$ 图像的 ISODATA 法提取水体的精度更高. 纵观 3 种方法, 基于 $N D W I$ 图像的 ISODATA 法提取的水体精度最高, 基于近红外波段的 ISODATA 法提取结果次之, NDWI 阈值法的提取效果最差. 研究结果对于离散型湖泊水体提取方法及数据源的选择等具有重要的借鉴和参考意义.
\end{abstract}

关键词: 遥感; 水体提取; NDWI; ISODATA; 鄱阳湖

\section{Accuracy comparison and analysis of methods for water area extraction of discrete lakes}

\author{
JI Hongxia ${ }^{1,2}$, FAN Xingwang ${ }^{1,2}$, WU Guiping ${ }^{1}$ \& LIU Yuanbo ${ }^{1}$ \\ (1: Nanjing Institute of Geography and Limnology, Chinese Academy of Sciences, Nanjing 210008, P. R. China) \\ (2: University of Chinese Academy of Sciences, Beijing 100049, P. R. China)
}

\begin{abstract}
Remote sensing is a widely used technology for water surface monitoring. Currently, there exists no general method for water surface extraction at a dispersed lake, which affects the accuracy of lake area monitoring. For investigation of different methods, this paper uses ALOS multi-spectral data to extract lake surface in Lake Poyang. The reference lake area is obtained by unsupervised classification ( ISODATA, Iterative Self-Organizing Data Analysis Technique Algorithm) based on fused 2. $5 \mathrm{~m}$ ALOS multi-spectral data. Subsequently, three methods are investigated based on $10 \mathrm{~m}$ ALOS data, including Normalized Difference Water Index $(N D W I)$ thresholding, NDWI-based ISODATA method and Near Infrared( NIR ) -based ISODATA method. Differences between these extractions are analyzed for intrinsic reasons. The main findings are: (1) all three methods can be used for water surface identification, ISODATA method provides more detailed information and larger water surface area; (2) NDWI-based method performs better than NIR-based method and the extraction accuracy increases; (3) NDWI-based ISODATA gets the most reliable result with $R^{2}=0.993$ and $R M S E=0.115 \mathrm{~km}^{2}$, followed by $N I R$-based ISODATA with $R^{2}=0.992$ and $R M S E=0.144 \mathrm{~km}{ }^{2}$, and $N D W I$ thresholding with $R^{2}=0.938$ and $R M S E=0.443 \mathrm{~km}^{2}$. The results provide important references for selection of method and data source for water extraction of lake in dry season.
\end{abstract}

Keywords: Remote sensing; water extraction; NDWI; ISODATA; Lake Poyang

湖泊是陆地表层系统各要素相互作用的节点, 与人类生产与生活息息相关, 在维系流域生态平衡、满足 生产生活用水和减轻洪旱灾害等方面发挥着不可替代的作用 ${ }^{[1]}$.湖泊面积是表征湖泊受气候变化与人类活 动影响程度的重要指示器, 实时准确地提取湖泊水面面积对于全面了解湖泊变化规律和演化趋势具有重要

* 国家重点基础研究发展计划“973”项目(2012CB417003) 和中国科学院南京地理与湖泊研究所“一三五”战略发展 规划项目 (NIGLAS2012135001) 联合资助. 2014-04-10 收稿;2014-07-02 收修改稿. 吉红霞 ( 1989 ), 女, 硕士 研究生; E-mail: jihongxia1026@163.com.

** 通信作者;E-mail: ybliu@ niglas. ac. cn. 
意义 ${ }^{[2]}$. 传统的湖泊面积测量方法大多是基于大比例尺测绘来实现的, 这种方法不但费时费力, 而且很难在 较大时空范围内开展. 遥感以其宏观、连续、实时等优势在对地观测中发挥着日益重要的作用 ${ }^{[3]}$. 利用卫星 遥感影像数据快速、准确地提取水体信息, 已经成为水资源调查和水资源宏观监测及湿地保护的重要 手段 ${ }^{[4]}$.

目前, 利用卫星遥感数据进行水体提取得到了广泛的应用,用于水体提取的遥感数据和方法多种多样. 譬如, Barton 等 ${ }^{[5]}$ 、杜云艳等 ${ }^{[6]}$ 提出了针对 NOAA (National Oceanic and Atmospheric Administration)/AVHRR (Advanced Very High Resolution Radiometer) 数据的水体提取方法, 认为近红外波段对水体较为敏感, 能较好 地用于提取水体. 吴赛等 ${ }^{[7]}$ 、丁莉东等 ${ }^{[8]}$ 利用 MODIS (Moderate Resolution Imaging Spectroradiometer) 数据, 实 现水体信息的自动提取, 主要有谱间关系法、单波段法及监督分类法. Jain 等 $^{[9]}$ 、Hui 等 $^{[10]}$ 基于更高分辨率 的 Landsat TM( Thematic Mapper) 数据提取水体信息,运用的方法包括单波段密度分割法、归一化差异水体指 数 ( Normalized Difference Water Index, NDWI) 阈值法、色度判别法、比率测算法、谱间关系法和非监督分类法 等. 此外还有一些国内外学者对其他卫星遥感数据提取水体进行了探讨 ${ }^{[11-15]}$.

波段比值法和非监督分类法是目前水体提取中应用最为广泛的两类方法 ${ }^{[16-17]}$. 其中, 波段比值法以 $N D W I$ 國值法最为常见, 主要是通过分析典型水陆交界处 $N D W I$ 图像的直方图分布特征, 配合确定的最优阈 值, 最终实现湖泊水面信息的提取 ${ }^{[18]}$. 非监督分类方法通过类内方差最小、类间方差最大的准则来进行分 类, 以 ISODATA (Iterative Self-organizing Data Analysis Technique Algorithm) 方法最为常用 ${ }^{[19]} . N D W I$ 方法具有 一定的物理基础, 对于连续型水体具有较好的适用性, 但是对于离散型湖泊水体而言, 例如湖、河、沼泽和滩 涂等共存的湿地, 在影像直方图中峰谷分布往往不够明晰, 因而该方法在整幅影像中很难确定最优阈值. ISODATA 法根据地物的光谱特征进行聚类, 仅需要确定初始的分类类别, 受主观因素的影响较小, 但采用不 同波段 (组合) 遥感数据进行非监督分类结果之间存在多大的差异尚未明晰. 由于季节性湖泊的水域面积随 水位波动变化剧烈, 例如洞庭湖和鄱阳湖, 在枯水期湖区分布着大片滩地, 水体分布支离破碎, 水面边界复 杂, 增大了水面提取的难度, 可能影响提取精度. 因此, 就目前水体提取中广泛采用的两种方法而言, 究竟哪 种方法对于离散型湖泊水体提取更为有效,是一个值得探讨的问题.

本文利用高分辨率 ALOS (Advanced Land Observation Satellite) PRISM ( Panchromatic Remote-sensing Instrument for Stereo Mapping) 数据和 AVNIR-2 (Advanced Visible and Near Infrared Radiometer Type 2) 数据, 以枯水期鄱阳湖子湖为研究对象, 将融合全波段图像非监督分类得到的 $2.5 \mathrm{~m}$ 高分辨率水体面积作为参考 值, 比较 $N D W I$ 阈值法和非监督分类法所提取水面面积之间的差异, 以及 $N D W I$ 非监督分类和近红外波段 ( Near Infra-Red, NIR) 非监督分类法所提取水面面积之间的差异, 并探讨产生差异性的原因,为有效提取离 散型湖泊水体提供方法论指导.

\section{1 研究区与数据预处理}

\section{1 研究区概况}

鄱阳湖地处江西省北部, 长江中下游南岸 (图 1), 是我国第一大淡水湖. 鄱阳湖是一个典型的季节性吞 吐型湖泊, 其承纳赣江、抚河、信江、饶河、修水五大支流来水, 经调蓄后由湖口注人长江, 与五河和长江之间 密切相关的水量交换关系, 形成了鄱阳湖独特的水情特征: 丰水期水面面积可达 $3000 \mathrm{~km}^{2}$, 而枯水期则不足 $1000 \mathrm{~km}^{2}$, 呈现出 “洪水一片、枯水一线” 的景观 ${ }^{[20]}$. 自 3 月下旬湖泊水位开始抬升, 7 月份达到最高水位, 至 10 月开始稳定下降, 至次年 1-2 月水位降至最低点 ${ }^{[21]}$. 在退水期间鄱阳湖水体分布零散, 湖区各种子湖泊 开始出露, 此外还分布有少量的养殖场, 类似的封闭离散水域十分常见. 但由于湖、沼泽、滩涂等共存, 水面 边界复杂, 增大了水面提取的难度.

\section{2 数据源及预处理}

ALOS 卫星发射于 2006 年, 搭载有全色遥感立体测绘仪 (PRISM)、可见光与近红外辐射计 (AVNIR-2) 和相控阵型 L 波段合成孔径雷达 (PALSAR) 3 种传感器. 其中多光谱 AVNIR-2 传感器包括了 4 个波段数据 的接收, 分别为蓝波段 $(0.42 \sim 0.50 \mu \mathrm{m})$ 、绿波段 $(0.52 \sim 0.60 \mu \mathrm{m})$ 、红波段 $(0.61 \sim 0.69 \mu \mathrm{m})$ 和近红外波段 $(0.76 \sim 0.89 \mu \mathrm{m})$, 其空间分辨率为 $10 \mathrm{~m}$. 全色 PRISM 传感器只有一个波段 $(0.52 \sim 0.77 \mu \mathrm{m})$, 空间分辨率 


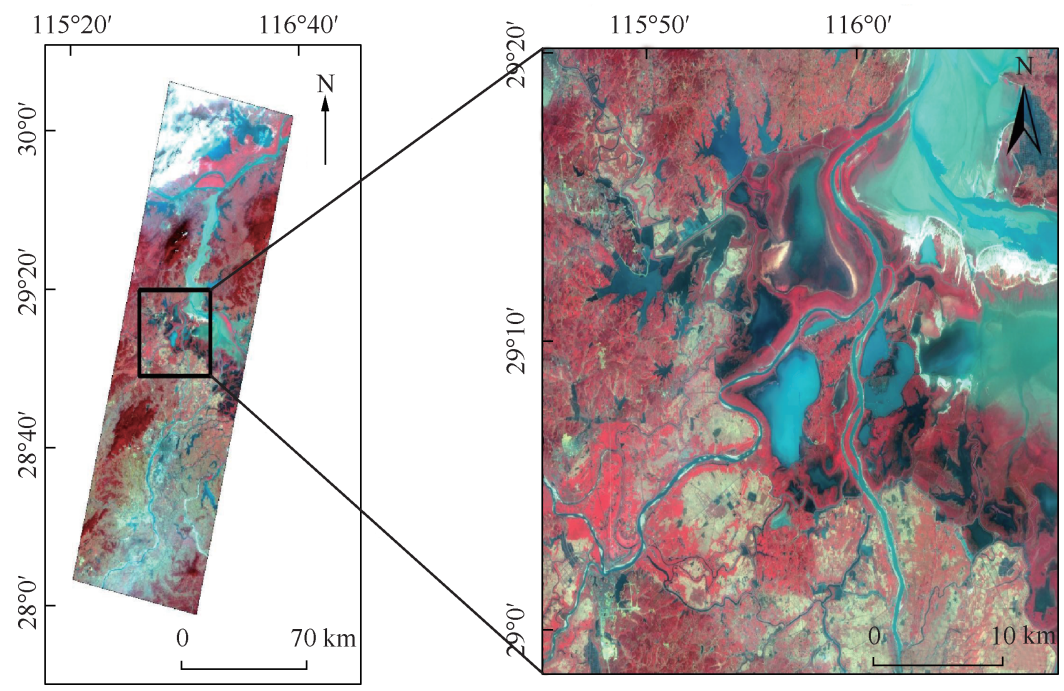

图 1 研究区位置

Fig. 1 Geographic location of study area

为 $2.5 \mathrm{~m}$. 本文选用 2007 年 10 月 24 日的 ALOS PRISM 全色波段数据和 AVNIR-2 多光谱波段数据. 该时期 鄱阳湖处于枯水期,湖、河、沼泽、滩涂等共存,具有十分复杂离散的水域分布形态.

ALOS 数据预处理主要包括噪声消除、图像融合与封闭水域轮廓的勾绘. 噪声消除是为了消除遥感影像 中的条纹、亮线及斑点等对水体提取的影响, 采用空间滤波的方法. 图像融合是指通过融合全色波段和多光 谱波段数据, 获取兼具高空间分辨率和多光谱分辨率的遥感数据. ALOS 卫星全色影像具有较高的空间分辨 率 $(2.5 \mathrm{~m})$, 多光谱影像具有较丰富的光谱信息, 光谱分辨率高, 通过将全色遥感影像与多光谱影像数据融 合, 提高影像的空间分辨率和光谱分辨率, 可以增强影像判读的准确性, 提高数据的使用效率 ${ }^{[22]}$. GramSchmidt 融合法 (简称 GS 变换) 是适合高分辨率影像的图像融合方法, 它可以很好地继承高分辨率影像的空 间分辨率, 而且保持光谱特性与原始多光谱影像最为接近, 光谱信息保存完好 ${ }^{[23]}$. 因而本文利用 GS 变换对 ALOS PRISM 全色波段数据和 AVNIR-2 多光谱数据进行融合得到 $2.5 \mathrm{~m}$ 高分辨率影像. 封闭水域轮廓的勾 绘采用手动方法, 结合野外调查, 通过人机交换确定 35 个封闭水域的边界, 据此可计算不同方法提取的水 体面积. 本研究中野外实际调查采用 GPS 差分测量中的 CORS 差分模式, 其定位精度较高, 达到了厘米级, 因而结合该结果可以保证数据的准确性. 实际调查也在枯水期进行, 但日期与影像日期不一致, 由于本文主 要对比不同水体提取方法的精度,因而不会影响研究结果.

本研究参考值的获取是利用 ISODATA 法对融合后 $2.5 \mathrm{~m}$ 高分辨率影像进行分类提取. 融合后的影像具 有 4 个光谱波段: 蓝波段 $(0.42 \sim 0.50 \mu \mathrm{m})$ 、绿波段 $(0.52 \sim 0.60 \mu \mathrm{m})$ 、红波段 $(0.61 \sim 0.69 \mu \mathrm{m})$ 和近红外波 段 $(0.76 \sim 0.89 \mu \mathrm{m})$, 空间分辨率为 $2.5 \mathrm{~m}$, 与原始高光谱波段相比具有更高的空间分辨率; 与原始全色影 像相比, 具有更高的光谱分辨率; 因而该影像增强了解译能力, 可提供更可靠的分析结果, 采用该影像提取 水体具有更高的精度 ${ }^{[24]}$. 结合野外实际调查, 初始分类类别设为 10 类, 最终通过目视对比与类别合并得到 所有水体并计算水体面积, 将该方法提取的水体面积作为其他方法的参考值.

\section{2 水体提取方法与比较}

\section{2. $1 N D W I$ 阈值法}

McFeeters ${ }^{[25]}$ 基于绿波段与近红外波段,提出 $N D W I$,表达式如下:

$$
N D W I=\frac{G-N I R}{G+N I R}
$$


式中, $G$ 和 $N I R$ 分别代表绿波段和近红外波段反射率. 在理想情况下, $N D W I$ 为正值时表示地面有水、雨雪覆 盖; $N D W I$ 等于 0 时表示地面覆盖为岩石或裸土等; $N D W I$ 为负值时表示有植被覆盖. 但实际情况下由于受到 水体表面植被等多种影响, 区分水体与其他地物的阈值往往不为零.

阈值法提取水体主要是根据不同地物之间灰度值的差异, 根据 NDWI 图像的直方图确定适当的阈值区 分水体与非水体. 阈值的选取是一个关键而难解决的问题. 以往研究发现, 水体阈值随影像的变化而变化, 因而需要根据具体的研究区域对每个时期的影像分别确定合适的阈值 ${ }^{[26]}$, 本文在具体确定阈值的过程中, 主要是基于不同地物之间灰度值的差异, 根据 $N D W I$ 直方图人机交互的分析方式加以确定 ${ }^{[27]}$. 通常情况下, $N D W I$ 图像的直方图呈现双峰的分布形态, 为了得到最优的阈值, 首先基于直方图中水体与非水体的波谷位 置中的点确定水体提取的最初阈值 ${ }^{[28]}$. 在此基础上, 通过不断调整阈值的大小直到提取出的水体与湖岸及 原始影像上的水体分布达到最佳匹配, 最终确定最优的阈值 ${ }^{[29]}$. 本研究中根据 $N D W I$ 图像的直方图分布, 并 结合 $2.5 \mathrm{~m} \mathrm{ALOS}$ 多光谱影像目视解译,通过反复比对,最终确定水体提取阈值为 0.336 .

\subsection{ISODATA 法}

ISODATA 法 (Iterative Self-Organizing Data Analysis Technique Algorithm, 迭代自组织数据分析算法), 是 通过引人参数而不断进行分裂与合并的非监督分类算法 ${ }^{[30]}$. 它通过调整样本所属类别完成样本的聚类分 析, 并使用类别的合并和分裂机制, 当某两类聚类中心距离小于某一阈值时, 将它们合并为一类, 当某类标 准差大于某一阈值或其样本数目超过某一阈值时, 将其分为两类. 在某类样本数目少于某阈值时, 需将其取 消. 如此, 根据初始聚类中心和设定的类别数目等参数迭代, 最终得到一个比较理想的分类结果 ${ }^{[31]}$. ISODA$\mathrm{TA}$ 法分类时一般需设置分类的数目比最终分类数量多 $3 \sim 4$ 倍, 再进行合并防止错分漏分, 以提高分类 精度 ${ }^{[32]}$.

\section{3 NDWI-ISODATA 法}

$N D W I$ 指数可以突出影像中的水体信息, 并能最大程度抑制植被信息 ${ }^{[33]}$. 对 $N D W I$ 图像采用 ISODATA 法进行分类可以避免人为确定阈值的过程. 根据影像上的地物类别数以及野外实际调查资料, 将初始类别 设为 10 类, 然后经过目视合并处理后, 提取出水体. 该分类结果可与 $N D W I$ 阈值法进行对比, 分析阈值不同 所导致分类结果存在差异的具体原因.

\subsection{NIR-ISODATA 法}

水体在近红外波段具有较低的光谱反射率, 能够很好地区分水体和非水体. 利用该波段进行 ISODATA 分类主要是为了与 NDWI-ISODATA 法形成对比, 分析原始数据不同对分类结果的影响. 参照以上 NDWIISODATA 法的参数设置, 初始类别数也设为 10 类, 然后对分类结果对照野外调查资料以及原始影像进行合 并处理, 从而提取出水体.

\section{5 对比分析}

对不同方法提取结果的比较包括定性比较和定量比较两方面. 定性比较主要是通过目视解译结合野外 实际调查结果比较不同方法的提取效果. 定量比较则是通过计算不同方法提取水体的面积并统计面积的总 值、最大值、最小值、平均值以及标准差等特征, 然后进行比较. 研究中将 $2.5 \mathrm{~m}$ 高分辨率融合影像 ISODATA 分类的结果作为参考值, 主要比较其他 3 种方法 ( NDWI 阈值法、NDWI-ISODATA 法和 NIR-ISODATA 法) 与 参考值的差别以及 3 种方法结果之间的差别. NDWI 阈值法和 $N D W I$-ISODATA 法均是在 NDWI 图像基础上 进行的, 因而可比较不同方法的提取结果. NDWI-ISODATA 法和 NIR-ISODATA 法采用相同的 ISODATA 法, 但初始数据不同, 因而可归为不同数据之间的提取结果比较. 在比较过程中, 主要利用了数理统计分析 方法.

\section{3 结果与讨论}

\section{1 水体提取结果}

分别利用高分辨率融合影像 ISODATA 法、NIR-ISODATA 法、NDWI-ISODATA 法和 NDWI 阈值法进行水 体提取, 得到的结果如图 2 所示. 可以得出: (1) 整体上利用 $2.5 \mathrm{~m}$ 高分辨率融合影像 ISODATA 法提取的水 体面积最大, $N D W I$ 阈值法提取的水体面积最小, NIR-ISODATA 法和 NDWI-ISODATA 法提取的水体面积相 
似, 存在微小差异; (2) $2.5 \mathrm{~m}$ 高分辨率融合影像 ISODATA 法提取得到的细小水体最多最密, NIR-ISODATA 法和 NDWI-ISODATA 法提取水体的微小信息量较多而密, NDWI 國值法对微小水体提取有一定的限制, 说明 ISODATA 法比阈值法更能反映水体的细节信息, 对于支离破碎的水体提取准确度更高; (3) 在水陆交界处, $N D W I$ 國值法提取的水体明显较少, NIR-ISODATA 法和 NDWI-ISODATA 法提取的结果则基本与实际符合, 因此 NIR-ISODATA 法和 NDWI-ISODATA 法可能对水陆交界处水体的提取效果更好; (4) 综合分析图中明 显水体信息, 提取的目视效果最优且稳定的是 NIR-ISODATA 法和 NDWI-ISODATA 法, NDWI 阈值方法效果 相对较差.
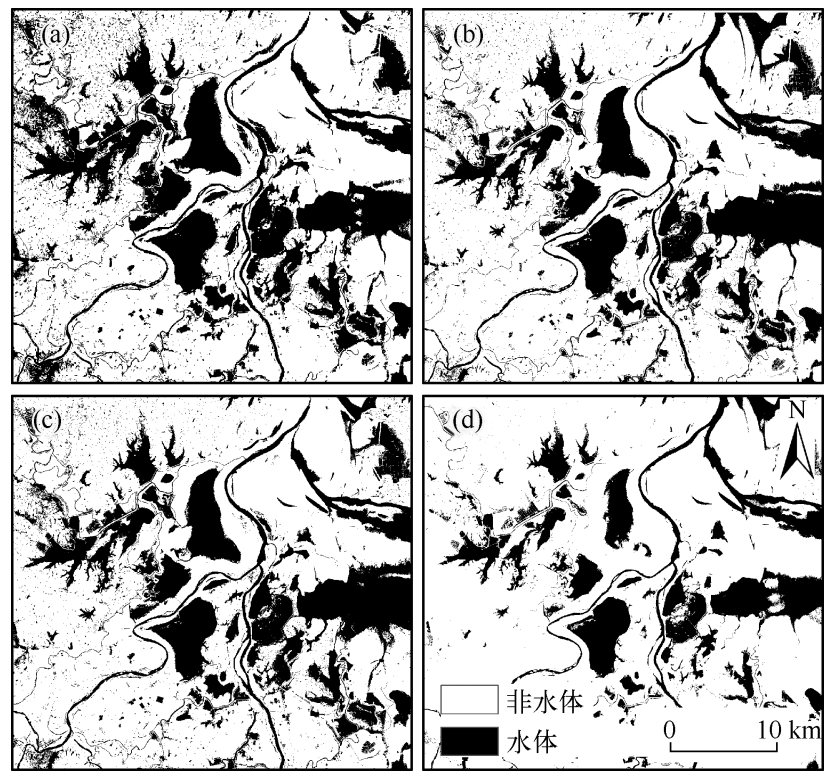

图 2 研究区内 4 种方法提取水体结果

（a:2. $5 \mathrm{~m}$ 高分辨率融合影像 ISODATA 法; b:NIR-ISODATA 法; $\mathrm{c}: N D W I$-ISODATA 法; $\mathrm{d}$ : $N D W I$ 阈值法）

Fig. 2 Results of water extraction of different methods in study area

( a: ALOS multi-spectral data; b: NIR-ISODATA; c: NDWI-ISODATA; d: NDWI)

\section{2 不同方法提取结果比较}

为了比较不同方法的提取结果, 对研究区内 35 个封闭水域分别利用以上 4 种研究方法进行提取, 获得 水面面积的统计特征, 包括总面积、最大面积、最小面积、平均面积以及标准差. NDWI-ISODATA 法和 NIRISODATA 法所提取的水体总面积分别为 158.97 和 $154.50 \mathrm{~km}^{2}$, 最大面积分别为 25.58 和 $25.18 \mathrm{~km}^{2}$, 最小 面积均为 $0.11 \mathrm{~km}^{2}$, 平均面积分别为 4.54 和 $4.41 \mathrm{~km}^{2}$, 标准差分别为 7.05 和 $6.85 \mathrm{~km}^{2} . N D W I$ 國值法所提 取的水体总面积为 $119.74 \mathrm{~km}^{2}$, 其最大值、最小值以及平均值分别为 $23.51 、 0.03 、 3.87 \mathrm{~km}^{2}$, 标准差为 $5.65 \mathrm{~km}^{2}$. 可以看出, 与作为参考值的融合影像提取结果相比, 3 种方法提取的水体总面积、最大面积、最小 面积以及平均面积均偏小, 而 $N D W I$ 國值法提取的面积最小, $N D W I$-ISODATA 法和 NIR-ISODATA 法的提取 结果非常接近,但 $N D W I$-ISODATA 法提取水体面积的标准差更接近于参考值 (表 1).

$N D W I$ 阈值法、NDWI-ISODATA 法和 NIR-ISODATA 法提取的水体面积与参考值之间呈显著正相关, 斜 率分别为 $0.7495 、 0.9607 、 0.9333$, 截距分别为 $-0.1498 、-0.0352 、-0.0325 \mathrm{~km}^{2}$, 可见 $N D W I$ 國值法提取结 果整体偏小, 而其他两种方法与参考值相差不大. 3 种方法提取水体的面积值与参考值拟合的确定系数 $R^{2}$ 分别为 $0.942 、 0.993$ 和 0.992 , 说明 NDWI-ISODATA 法的提取结果与参考值的拟合效果最好. 均方根误差 $(R M S E)$ 分别为 $0.443 、 0.115 、 0.144 \mathrm{~km}^{2}$, 表明 3 种方法提取水体精度由高到低依次为 $N D W I$-ISODATA 法、 NIR-ISODATA 法和 NDWI 阈值法 ( 图 3 ). 
表 1 不同方法提取水体面积统计

Tab. 1 Statistics of water surface areas derived from different methods

\begin{tabular}{cccccc}
\hline 方法 & 总面积 $/ \mathrm{km}^{2}$ & 最大面积 $/ \mathrm{km}^{2}$ & 最小面积 $/ \mathrm{km}^{2}$ & 平均面积 $/ \mathrm{km}^{2}$ & 标准差 $/ \mathrm{km}^{2}$ \\
\hline 融合影像 ISODATA 法 & 163.16 & 26.15 & 0.12 & 4.76 & 7.31 \\
NDWI-ISODATA 法 & 158.97 & 25.58 & 0.11 & 4.54 & 7.05 \\
NIR-ISODATA 法 & 154.50 & 25.18 & 0.11 & 4.41 & 6.85 \\
$N D W I$ 阈值法 & 119.74 & 23.51 & 0.03 & 3.87 & 5.65 \\
\hline
\end{tabular}

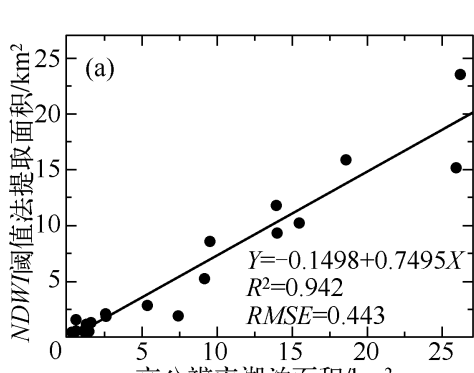

高分辨率湖泊面积 $/ \mathrm{km}^{2}$

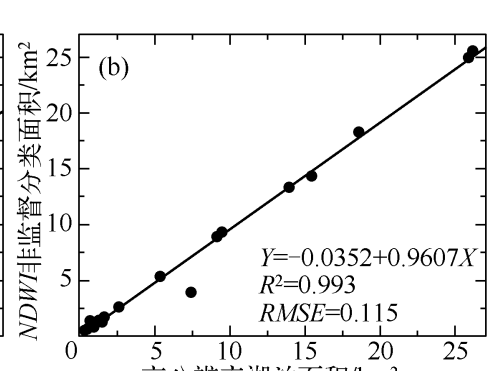

高分辨率湖泊面积 $/ \mathrm{km}^{2}$

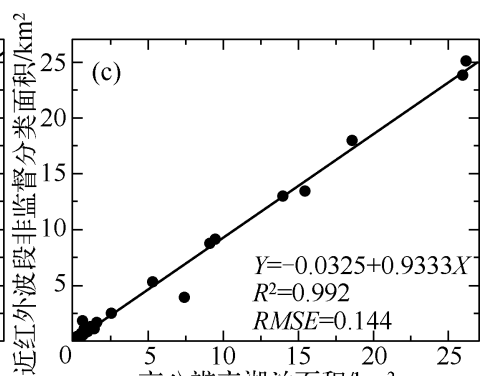

高分辨率湖泊面积 $/ \mathrm{km}^{2}$

图 3 不同方法提取水体面积的精度 ( $\mathrm{a}: N D W I$ 阈值法; $\mathrm{b}: N D W I$-ISODATA 法; $\mathrm{c}: N I R$-ISODATA 法)

Fig. 3 Accuracy of water surface areas derived from different methods

( a: NDWI; b: NDWI-ISODATA; c: NIR-ISODATA)

\section{3. $3 N D W I$ 阈值法与 NDWI-ISODATA 法的比较}

虽然 $N D W I$ 阈值法与 $N D W I$-ISODATA 法都使用 $N D W I$ 图像作为分类基础,但两者之间存在着分类差异. $N D W I$-ISODATA 法与 $N D W I$ 阈值法所提取结果之间的斜率为 1.2215 , 截距为 $0.3631 \mathrm{~km}^{2}, R^{2}$ 为 $0.957, R M S E$ 为 $0.372 \mathrm{~km}^{2}$, 表明 $N D W I$-ISODATA 法普遍大于 $N D W I$ 阈值法的提取结果 (图 4a). $N D W I$ 阈值法提取面积为 参考值的 74.95\%, NDWI-ISODATA 法提取的水体面积为参考值的 $96.07 \%$, 因而前者比后者大 $21.12 \%$. 造 成差异的主要原因在于 ISODATA 法采用迭代方法,不涉及人为选取阈值的过程,分类结果更为客观.

为进一步识别两种方法之间的差异性来源, 对所有 NDWI-ISODATA 法所提取水体对应的阈值进行统 计, 发现这些阈值均在 0.2 左右, 小于 $N D W I$ 阈值法提取水体时所确定的阈值 0.336 , 阈值越小所提取的水 体面积越大. 利用 $N D W I$ 阈值法, 将阈值设置为 0.2 , 重新提取水体. 结果显示, 当阈值设为 0.2 时, $N D W I$ 阈 值法与 NDWI-ISODATA 法提取的水体面积非常相近, 拟合 $R^{2}=1, R M S E=0.003 \mathrm{~km}^{2}$ (图 4b). 这些分析表 明, NDWI-ISODATA 法相对 NDWI 阈值法来说, 避免了人为确定阈值的偶然性和随机性, 提取的结果更准确.
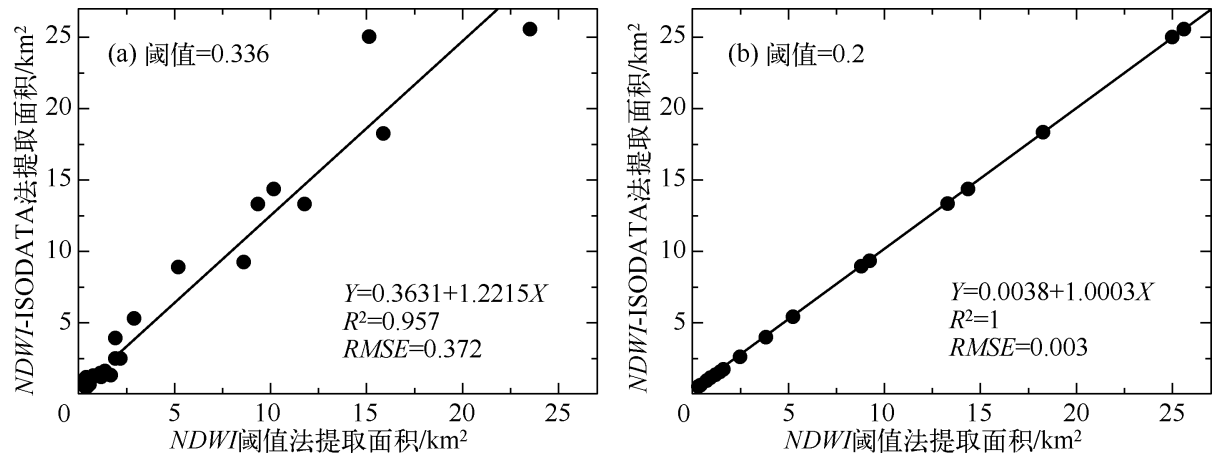

图 4 基于 NDWI 阈值法与 ISODATA 法提取水体结果的关系

Fig. 4 Relationship between water surface areas derived from NDWI thresholding and NDWI-based ISODATA methods 


\section{4 NDWI-ISODATA 法与 NIR-ISODATA 法的比较}

NDWI-ISODATA 法与 NIR-ISODATA 法虽然使用 了不同波段的数据,但两者所提取的水体面积非常接 近. 这两种方法所提取的水体面积呈显著的线性相关, $R^{2}=0.999, R M S E=0.047 \mathrm{~km}^{2}$, 拟合偏差 Bias $=$ $-0.0001 \mathrm{~km}^{2}$. 线性拟合直线的斜率为 1.0285 , 截距为 $0.0021 \mathrm{~km}^{2}$ (图 5 ), 表明 NDWI-ISODATA 法所提取的 水体面积略大于 NIR-ISODATA 法. 与参考值相比, NDWI-ISODATA 法与 NIR-ISODATA 法的 RMSE 分别 为 0.115 和 $0.144 \mathrm{~km}^{2}$, 说明 $N D W I$-ISODATA 法所提取 结果更准确一些. 总体而言, 这两种方法均为 ISODATA 法, 不同之处在于数据源, 一个使用近红外波段数据, 另一个使用 $N D W I$ 数据. $N D W I$ 数据是将近红外波段数 据和绿波段数据进行组合处理得到的, 相对于单波段 近红外波段数据而言, 它综合了两个波段的水体光谱 特征,包含了更多的水体信息, 其水体识别精度比单波 段高.

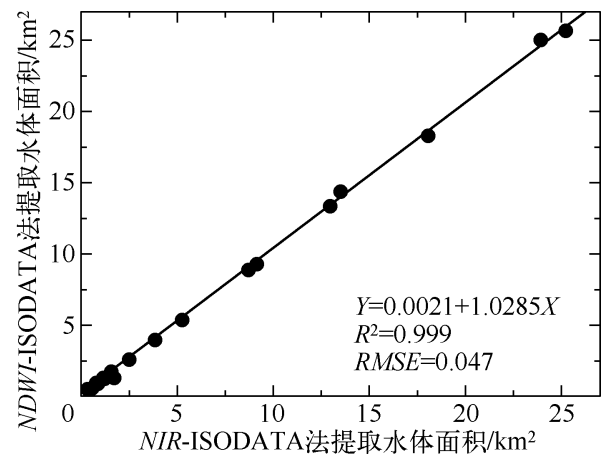

图 5 NDWI-ISODATA 法与 NIR-ISODATA 法提取水体结果比较

Fig. 5 Comparison between water surface areas derived from $N D W I$-based ISODATA and NIR-based ISODATA methods

\section{4 结论}

本文以鄱阳湖湖区为研究对象, 以 ALOS PRISM 全波段数据和 AVNIR-2 多光谱数据为基础, 首先将 PRISM 数据和 AVNIR-2 数据进行影像融合, 得到 $2.5 \mathrm{~m}$ 高分辨率影像并对融合影像采用 ISODATA 法进行 水体提取, 将提取的 $2.5 \mathrm{~m}$ 分辨率水面面积作为参考值. 然后分别使用 NDWI 阈值法、NDWI-ISODATA 法和 NIR-ISODATA 法提取 AVNIR-2 多光谱数据, 得到了 $10 \mathrm{~m}$ 分辨率的水体分布. 在此基础上分析了不同方法提 取结果之间的差异性及产生原因,得到以下研究结论:

1) $N D W I$ 國值法与 NDWI-ISODATA 法都使用 NDWI 图像作为分类基础, 但两者之间存在着分类差异. 两 种方法提取的水体面积相对参考值的斜率分别为 0.7495 和 0.9607 , 截距分别为 -0.1498 和 $-0.0352 \mathrm{~km}^{2}$, $R^{2}$ 值分别为 0.942 和 $0.993, R M S E$ 值分别为 0.443 和 $0.115 \mathrm{~km}^{2}$. 将两种方法对应的水体面积作线性拟合后 得到的线性斜率为 1.2215 , 截距为 $0.3631 \mathrm{~km}^{2}$, 拟合 $R^{2}=0.957, R M S E=0.372 \mathrm{~km}^{2}$, 说明 $N D W I$-ISODATA 法 的提取效果优于 $N D W I$ 阈值法. 改变最优阈值后, $N D W I$ 阈值法与 $N D W I$-ISODATA 法的提取结果几乎相同. $N D W I$-ISODATA法相对 NDWI 阈值法避免了阈值选取的随机性,更能准确地反映水体的细节信息.

2) NDWI-ISODATA 法与 NIR-ISODATA 法虽然使用了不同波段的数据, 但两者所提取的水体面积非常 接近. 两种数据提取的水体面积相对参考值的斜率分别为 0.9607 和 0.9333 , 截距分别为 -0.0352 和 $-0.0325 \mathrm{~km}^{2}, R^{2}$ 值分别为 0.993 和 $0.992, R M S E$ 值分别为 0.115 和 $0.144 \mathrm{~km}^{2}$, 两者的 $R^{2}$ 值相似, 但 $R M S E$ 差异较大. 对两种结果作线性拟合的结果显示 NDWI-ISODATA 法的提取效果优于 NIR-ISODATA 法. 相对近 红外单波段,多波段组合指数 NDWI增强了水体提取信息,提高了水体提取准确性.

3 ) 综合比较发现, 3 种方法均可以较好地提取出图像中的主体水体区域. 在细小水体提取方面, NDWIISODATA 法的提取结果最好, NIR-ISODATA 法的提取效果其次, $N D W I$ 阈值法的提取效果最差. 3 种水体提 取效果从好到差依次是: NDWI-ISODATA 法、NIR-ISODATA 法和 NDWI 阈值法. NDWI-ISODATA 法不仅综合 了多个波段的水体光谱特征, 同时不需要进行水体國值的选取, 避免了阈值选择所带来的主观误差, 更能高 精度地提取水体. 因而认为基于 NDWI 数据的 ISODATA 法可以作为针对枯水期离散型湖泊水体提取精度较 好的一种普适性的方法.

\section{5 参考文献}

[ 1 ] 李景刚,李纪人,黄诗峰等. Terra/MODIS 时间序列数据在湖泊水域面积动态监测中的应用研究. 自然资源学报, 
$2009,24(5): 923-933$.

[ 2 ] Huang S, Li J, Xu M. Water surface variations monitoring and flood hazard analysis in Dongting Lake area using long-term Terra/MODIS data time series. Natural Hazards, 2012, 62 (1) : 93-100.

[3] 李景刚, 李纪人, 黄诗峰等. 近 10 年来洞庭湖区水面面积变化遥感监测分析. 中国水利水电科学研究院学报, 2010,8 (3) :201-207.

[4] 邓劲松, 王 珂, 邓艳华等. SPOT-5 卫星影像中水体信息自动提取的一种有效方法. 上海交通大学学报:农业科学 版,2005,23(2):198-201.

[ 5 ] Barton IJ, Bathols JM. Monitoring floods with AVHRR. Remote Sensing of Environment, 1989, 30(1): 89-94.

[6] 杜云艳,周成虎. 水体的遥感信息自动提取方法. 遥感学报, 1998,2(4):264-268.

[ 7 ] 吴 赛, 张秋文. 基于 MODIS 遥感数据的水体提取方法及模型研究. 计算机与数字工程,2005,33(7):1-4.

[8] 丁莉东, 吴 吴. MODIS 图像湖泊水体信息的快速识别与制图. 海洋测绘,2006,26(6):31-34.

[ 9 ] Jain S, Singh R, Jain M et al. Delineation of flood-prone areas using remote sensing techniques. Water Resources Management, $2005, \mathbf{1 9}(4)$ : 333-347.

[10] Hui F, Xu B, Huang H et al. Modelling spatial-temporal change of Poyang Lake using multitemporal Landsat imagery. International Journal of Remote Sensing, 2008, 29(20): 5767-5784.

[11] 席晓燕, 沈 楠, 李小娟. ETM + 影像水体提取方法研究. 计算机工程与设计,2009, (4):993-996.

[12] 杨存建,周成虎. 利用 RADARSAT SWA SAR 和 LANDSAT TM 的互补信息确定洪水水体范围. 自然灾害学报, $2001, \mathbf{1 0}(2): 79-83$.

[13] 都金康,黄永胜. SPOT 卫星影像的水体提取方法及分类研究. 遥感学报,2001,5(3):214-219.

[14] 何智勇, 章孝灿, 黄智才等.一种高分辨率遥感影像水体提取技术. 浙江大学学报: 理学版,2005,31(6):701-707.

[15] 贾德伟,钟仕全, 李 雪等. 环境一号卫星高光谱数据水体信息提取方法. 测绘科学, 2011,36(4):128-130.

[16] 宋 平,刘元波, 刘燕春. 陆地水体参数的卫星遥感反演研究进展. 地球科学进展, 2011,26(7):731-740.

[17] 李文庆,姜琦刚,邢 宇等. 基于 Google Earth 的 ETM + 遥感图像自动分类方法. 江西农业学报,2012,24 (12): $158-163$.

[18] 曲 伟,路京选,李 琳等. 环境减灾小卫星影像水体和湿地自动提取方法研究. 遥感信息, 2011,(4):28-33.

[19] 邓书斌. ENVI 遥感图像处理方法. 北京:科学出版社,2010.

[20] 王苏民,窦鸿身. 中国湖泊志. 北京:科学出版社, 1998 .

[21] 杨菁媛,方朝阳,陈晓玲. 基于多时相 ENVISAT ASAR 的鄱阳湖水面覆盖信息提取及其应用研究. 安徽农业科学, 2011,39 ( 11 ):6874-6876.

[22] 戴丽君,刘 闯. ALOS 融合影像中水体信息提取方法研究. 水资源与水工程学报,2011,22(3):106-109.

[23] 赵 红,贾永红,张晓萍等. 遥感影像融合方法在 ALOS 影像水体信息提取中的应用研究. 水资源与水工程学报, 2010,21 (3) : $56-58$.

[24] Jin X, Davis CH. An integrated system for automatic road mapping from high-resolution multi-spectral satellite imagery by information fusion. Information Fusion, 2005, 6(4) : 257-273.

[25] McFeeters SK. The use of the Normalized Difference Water Index ( NDWI) in the delineation of open water feature. International Journal of Remote Sensing, 1996, 17(7) : 1425-1432.

[26] Ming-Guo MA. 基于遥感的湖泊水域动态变化监测研究进展. 遥感技术与应用,2009,24(5):674-684.

[27] 郭 鹏,邹春辉,赵学斌. MODIS 影像水体监测方法研究进展. 气象科技,2013,40(6):869-873.

[28] Bryant RG. Application of AVHRR to monitoring a climatically sensitive playa. Case study: Chott el Djerid, southern Tunisia. Earth Surface Processes and Landforms, 1999, 24(4) : 283-302.

[29] Liu Y, Song P, Peng J et al. A physical explanation of the variation in threshold for delineating terrestrial water surfaces from multi-temporal images: effects of radiometric correction. International Journal of Remote Sensing, 2012,33 ( 18 ) : $5862-5875$.

[30］李晓娟,宫兆玲,刘晓萌. ENVI 遥感影像处理教程. 北京: 中国科学环境出版社,2007.

[31] 杨小明,罗 云. ISODATA 算法的实现与分析. 采矿技术,2006,6(2):66.

[32] 薛星宇, 刘红玉. 基于 ALOS 影像的盐城海滨湿地遥感信息分类方法研究. 遥感技术与应用,2012,27(2):248-255.

[33] 严恩萍, 林 辉, 莫登奎. 基于 CIWI 模型的洪湖市水体识别及动态监测. 水生态学杂志,2011,32(6):14-19. 